\title{
Measurement Research of Motorized Spindle Dynamic Stiffness under High Speed Rotating
}

\author{
Xiaopeng Wang, Yuzhu Guo, and Tianning Chen \\ School of Mechanical Engineering, State Key Laboratory for Strength and Vibration of Mechanical Structures, \\ Xian Jiaotong University, Xian 710049, China
}

Correspondence should be addressed to Xiaopeng Wang; xpwang@mail.xjtu.edu.cn

Received 28 September 2014; Accepted 3 February 2015

Academic Editor: Roger Serra

Copyright (c) 2015 Xiaopeng Wang et al. This is an open access article distributed under the Creative Commons Attribution License, which permits unrestricted use, distribution, and reproduction in any medium, provided the original work is properly cited.

High speed motorized spindle has become a key functional unit of high speed machine tools and effectively promotes the development of machine tool technology. The development of higher speed and more power puts forward the stricter requirement for the performance of motorized spindle, especially the dynamic performance which affects the machining accuracy, reliability, and production efficiency. To overcome the problems of ineffective loading and dynamic performance measurement of motorized spindle, a noncontact electromagnetic loading device is developed. The cutting load can be simulated by using electromagnetic force. A new method of measuring force by force sensors is presented, and the steady and transient loading force could be measured exactly. After the high speed machine spindle is tested, the frequency response curves of the spindle relative to machine table are collected at $0 \sim 12000 \mathrm{rpm}$; then the relationships between stiffness and speeds as well as between damping ratio and speeds are obtained. The result shows that not only the static and dynamic stiffness but also the damping ratio declined with the increase of speed.

\section{Introduction}

High speed motorized spindle, which integrates machine tool spindle and motor to dispense with belt transmission and gear transmission, effectively promotes the development of machine tool technology. High speed motorized spindle has become a key functional unit of high speed machine tools, and its performance effects the development of machine tools. In recent years, the rotating speed of high speed spindle has reached hundreds of thousands, and the $d N$ value (the $d N$ value is the product of bearing inner diameter [mm] and working speed $[\mathrm{r} / \mathrm{min}]$, and it is an important parameter of bearings to describe the degree of high speed) has been approaching 2-3 millions. The development of higher speed and more power puts forward the stricter requirement for the performance of high speed spindle, especially the dynamic performance which affects the machining accuracy, reliability, and production efficiency. Therefore, the research on dynamic performance has been more important [1].

At present, the research of the static and dynamic performance of high speed spindle focused on theoretical modeling and finite element simulation. Wang and Chang developed a machine tool spindle-bearing system model based on finite element model (FEM); they analyzed the spindle dynamics by assuming the spindle as a Reyleigh beam. The stiffness and damping of the bearings were modelled by two constant matrices. However, the model did not include the effects of rotation and preload, so there were no centrifugal forces or gyroscopic moments [2]. In the model of Nelson and McVaugh, the FEM formulation of a rotor shaft was built using the Euler Bernoulli beam theory with considering the rotation; therefore the centrifugal forces and gyroscopic moments were included [3]. Then, Zorzi and Nelson extended the model of Nelson and McVaugh by adding the internal damping, while the bearings were represented as stiffness devices. And the model was developed with Timoshenko beam theory, which considers the effects of rotation and shear, so the models were more precise [4]. Xiong et al. developed a new dynamic milling model including a rotating spindle and the gyroscopic effect on the stability characteristics of the milling system for the first time. The way of combining a FEM representation and milling 
cutting force predicted that the gyroscopic moment would not change the instability regions in milling, but it changes the real part of the eigenvalues of the system or reduces the critical axial depth of cut [5]. Cao and Altintas presented a general method for modelling a spindle system, which consists of spindle shaft, angular contact ball bearings, and housing. The method could analyze the effect of centrifugal forces and gyroscopic moments of the spindle rotor and bearings, as well as the preload. The stiffness of the bearings, contact forces on bearing balls, natural frequencies, time history response, and frequency response functions can be obtained by applying the cutting forces to the spindle for a given preload, including the rotating effects of the spindle on both bearing stiffness and the dynamics of the spindle system. The method simulated different spindle speeds, and it was only verified in a static model. The results predicted that a centrifugal force has more influence on the natural frequency than the gyroscopic moment [6]. Gagnola et al. proposed a finite element model of a spindle system based on Timoshenko beam theory, which also included the effects of centrifugal forces and gyroscopic moments, and predicted the critical speed using the Campbell diagram, but have not considered the change of the bearing dynamics under high speed rotation [7]. Except for the pure dynamic analysis, the heat effects on spindle cannot be neglected. Lin et al. presented an integrated thermomechanical-dynamic model to characterize mechanical and thermal influences on the dynamic behaviors of motorized spindles during very high speed rotation. They focused on the bearing preload and high speed rotation effects on the bearing temperature rise and analyzed qualitatively that the bearing heat affected the bearing stiffness as well as overall spindle dynamic [8]. The thermomechanical-dynamic model developed by Li may be most comprehensive and complete, which consists of a comprehensive bearing dynamic model, a shaft dynamic model, and a thermal model. The integrated model can simulate accurately the bearing heat generation and thermal expansion of the whole system based on the bearing configuration, as well as other parameters effects on the natural frequency of spindle. The effects are illustrated through numerical analysis [9].

With the development of finite element technology, commercial FEM software has become an essential solution technology in the static, dynamic, and thermal analysis of spindle performance, which makes the analysis more efficiently. Rantatalo compared experimental testing with the FEM simulation in order to investigate the effect of the gyroscopic moment and the speed on eigenfrequency and modal shape of a high speed motorized spindle. The FEM simulations incorporated a spindle speed sensitive bearing stiffness, a separate mass and stiffness radius, and a stiffness radius sensitive shear deformation factor. The analysis showed that centrifugal force that acted on the bearing balls resulted in a softening of the bearing stiffness with increased spindle speed [10]. Li et al. studied the characteristics of mode and harmonic response of the high speed motorized spindle with the FEM. A dual-dimension model with the FEM is developed and simulated using ANSYS commercial software. Their research showed that dynamic displacement and stiffness do not change significantly compared with the static performance of working of the motorized spindle [11]. The similar method was employed in Liu's research, Liu et al. investigated the characteristics of a high speed motorized spindle system through taking into account bearing support contact interface, which is established by springdamper element COMBIN 14. The simulations of static, modal, harmonic response and thermal deformation are conducted by means of ANSYS commercial software [12]. Similarly, a Finite Element Analysis of a high speed spindle with variable bearing preload was presented to compare against the experimental data by Delgado et al. The spindle model was simulated and the effect of preload on stiffness and frequency response function (FRF) was analyzed using ANSYS and SpindlePro. The research showed that gyroscopic moment is effective at lower damping ratios and stiffness [13]. Holkup used ANSYS software to verify the prediction of a finite element method-based thermomechanical model of spindles with rolling bearings, which included the temperature distribution and thermal growth, together with transient changes in bearing stiffness and contact loads under specified operating conditions [14].

Compared with the theoretical modeling and FEM simulation, the experimental test can obtain directly the static and dynamic characteristic of the high speed spindle more accurately. On the other hand, these testing parameters would be helpful to the performance evaluation and the modeling modification of the spindle, which is very significant for improving the spindle performance. As a kind of testing method, the dry run test can reflect the performance of the spindle in some extent by testing the vibration and deformation of the spindle during operation. But the machining experiment may be more effective.

Gagnola et al. carried out a milling experiment on a motorized high speed milling spindle at 15,000 rpm in order to confirm the validity of model predictions. Experimental validation was performed on the basis of synchronous excitation from a five-tooth tool; the speed dependent vibration level of the tool body was recorded using a laser displacement sensor. The result showed that the stability lobe diagram generated from the nonrotating transfer function underpredicted the allowable depth of cut and sometimes led to an inaccurate stability limit prediction [15]. Zaghbani and Songmene developed a complete method for estimating the dynamic modal parameters of a machine tool system during machining using two methods of operational modal analysis (OMA). The stability lobes was generated using OMA and can predict more accurately the stability zones. They detected that the spindle speed and boundary conditions during machining operations influence the machine tool's dynamic parameters [16].

However, the problem of controlling and measuring loading force led to the difficulty in the dynamic performance of high speed spindle. Then, the method of loading test is developed to substitute the machining experiment. The cutting forces can be simulated using different methods of loading force to vibrate the spindle, and the frequency response function (FRF) that includes the parameters of natural frequencies, stiffness, and damping ratios will be 
obtained quickly by measuring force and displacement at the tool tip. The chief task of the loading test is how to solve the problem of loading.

Albrecht et al. measured the transfer functions of the spindle sensor system at different speeds using impacthammering on the outer bearing ring. The outer bearing ring is kept stationary while the spindle rotates, so that an impact force can be applied to the spindle [17]. The loading method of impact testing was also used by Ozturk et al. to measure the spindle-machine dynamics under variable preload. A standard artifact which was inserted in the spindle acted the loaded target, on which an instrumented hammer applied the force. An accelerometer was used to measure the response of the standard artifact. The tool tip FRF was obtained, which reflected the change of the spindlemachine dynamics under different preload and speed [18]. In a similar way, Cheng et al. used the impact testing to identify the at-speed spindle-machine dynamics. The difference was that the hammer impacted directly the rotating cylindrical standard artifact instead of an outer bearing ring. They described the prediction of rotating tool point frequency response functions using receptance coupling substructure analysis (RCSA), and the article provided the experimental validation and a predictive procedure for analyzing variation in stability with spindle speed [19]. Furthermore, J.-S Chen and K.-W Chen loaded the spindle using an active bearing loading monitoring and control mechanism that consisted of an integrated strain-gage load cells and piezoelectric actuators [20]. Tsuneyoshi developed a contact type loading device for the measurement of axial stiffness [21].

Because of the existing problems, such as vibration, friction, and noise, the contact loading is confined to the condition that spindle is static or low speed. Nevertheless, it is obvious that the test of spindle under high speed rotation is more accurate and more precise to reflect the actual operational performance of the spindle. So the noncontact loading method is adopted to meet the needs of high speed testing.

Feng et al. presented a noncontact gas-loading device for the static stiffness measurement of high speed spindles. The hydrostatic gas film formed between the loader and rotating spindle can apply loads upon a rotating spindle with noncontact condition [22]. However, the device could not apply dynamic force.

Compared with the contact loading method and gasloading, the noncontact electromagnetic loading is more efficient and easy to control.

Kwiatkowski [23] and Chowdhury et al. [24] presented more early the method of electromagnetic in order to allow the stiffness measurement of machine tool with the spindle rotating. Kwiatkowski designed the loading test platform and deduced the attractive force formula of the loading device and studied the influencing factors of the electromagnetic loading. Chowdhury et al. obtained a set of harmonic response loci by testing a milling machine using the electromagnetic exciter of Kwiatkowski. The comprehensive test showed that the experimental results were affected by spindle rotation, spindle speed, exciting force amplitude and preload, and so

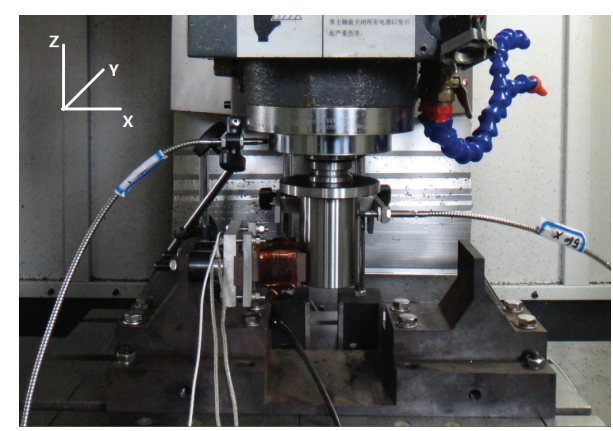

FIGURE 1: Electromagnetic loading experiment device.

forth. The reliable technique for determining the harmonic response loci from forced vibration tests was established. Yamazaki et al. [25] and Matsubara et al. [26] measured the high speed spindle stiffness in the condition that the spindle rotates at high speed by using a magnetic loading device. The eddy current which decreased the electromagnetic attractive force was analyzed by FEM modeling, and a circumferential grooved tool is designed and manufactured to cut down the influence of eddy current.

However, the devices of the above research cannot obtain loading force directly but calculate the force by the magnetic induction intensity measured by search coils in the magnet core. The method has a low precision and a complicated calibration, so it is not suitable for the stiffness test which needs the accurate measurement of loading force.

In the paper, a noncontact electromagnetic loading device is developed to meet the testing requirement for static and dynamic stiffness measurement of spindle during high speed rotating. A new method of measuring force by using force sensors is presented, so the steady and transient loading force can be measured exactly. The test is implemented on the high speed spindle of milling machine, which shows that the static and dynamic stiffness declined with the increase of speed.

\section{Loading and Measurement Device}

2.1. Summary of Noncontact Electromagnetic Device. According to the need of the measurement of high speed spindle stiffness, the noncontact electromagnetic loading and measurement device is designed in Figure 1.

Structure diagram of the loading and measurement device is shown in Figure 2.

The device is composed of four parts as follows:

(1) electromagnetic loading part: electromagnet and dummy tool,

(2) data acquisition and analysis part: German $m+p$ Vibpilot-8 data acquisition and analysis system (DAAS), computer,

(3) force and displacement measurement part: force sensors and displacement sensors,

(4) others: power amplifier and support table. 


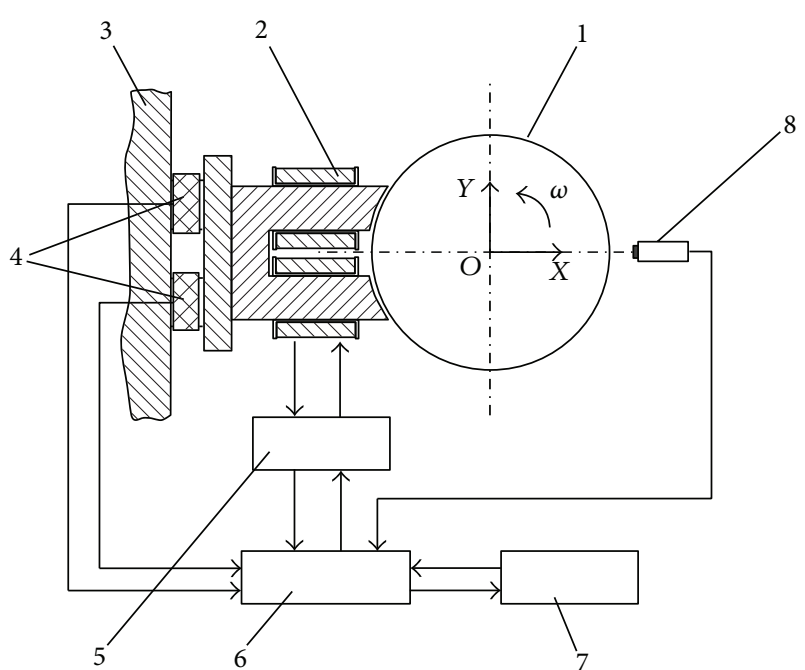
1: Dummy tool
2: Magnet loader
3: Support table
4: Force sensor
5: Power amplifier
6: Data acquisition and analysis system (DAAS)
7: Computer
8: Displacement sensor

FIgURE 2: Structure diagram of loading and measurement device.

TABLE 1: Parameter of electromagnet.

\begin{tabular}{lc}
\hline Parameters & Value \\
\hline Coil turns $n$ & 100 \\
Magnetic pole $S$ & $380 \mathrm{~mm}^{2}$ \\
Air gap $e$ & $0.50 \mathrm{~mm}$ \\
Maximum current $I$ & $4 \mathrm{~A}$ \\
\hline
\end{tabular}

The core of electromagnet is made by $0.35 \mathrm{~mm}$ silicon steel sheet, and the parameters are shown in Table 1.

The dummy tool that was designed to replace the standard tool holder and tool is composed of a BT30 tool holder and a loading cylinder. As a carrier, it can transmit the force from electromagnet to motorized spindle. The FRF testing results may be influenced by the different types of dummy tool [19]. However, the error is allowable, because the stiffness of dummy tool is enough large and the added mass is small.

The working principle of the device is as follows.

DAAS $(m+p$ Vibpilot- $8, m+p$ international Mess- und Rechnertechnik $\mathrm{GmbH}$ ) generates the required voltage signal, which is controlled by SO Analyzer 4.2 software in computer. The voltage signal will be amplified and transferred into a current signal, which inlets the coil of electromagnet. Electromagnet generates magnetic field flowing past dummy tool and the two poles of electromagnet. Dummy tool is applied alternating load in the magnetic field, which vibrated the spindle connected with dummy tool. The displacement response of the spindle is measured by displacement sensors, and the reacting force of tool countering electromagnet is measured by the strain type force sensors. Afterwards, the

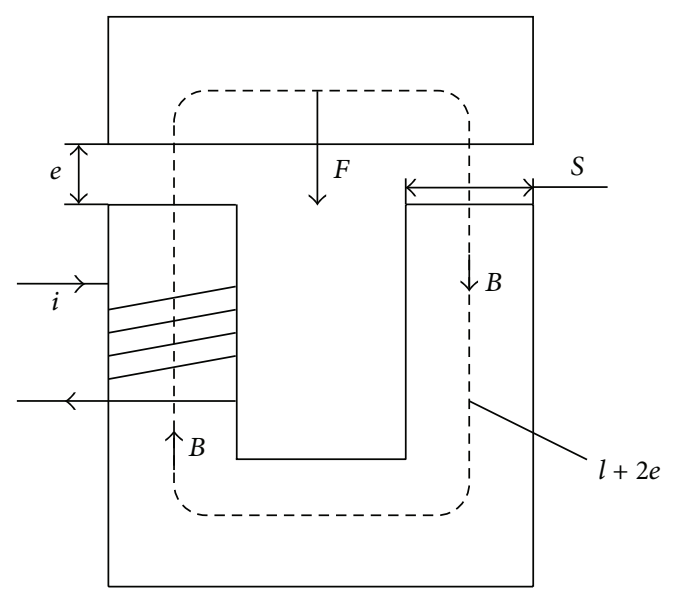

Figure 3: Construction of U-electromagnet.

FRF of the spindle is obtained by the data processing of force and displacement signal.

There are some advantages of the device as below.

(1) Noncontact loading is adopted, which is a more appropriate way for rotary spindle to avoid the friction, vibration, and noise of contact loading.

(2) It is easy to control the load by DAAS, which does not need manual adjustment. The load can be measured in real time by force sensors and fed back into DAAS.

(3) The dummy tool includes a standard tool holder to allow the installation and disassembly with different spindles.

2.2. The Principle of Loading and Measuring Force. Electromagnetic load is a method of using magnetic field controlled by current to excite ferromagnetic substance. The basic application is electromagnet shown as Figure 3.

Because of the resemblance to active magnetic bearing in structure and principle, the electromagnetic force of the device (Figure 1) can be derived by the Maxwell equation in electromagnetic field theory. When the nonsaturated magnetic field is distributed uniformly throughout the air gap between electromagnet and tool and the influence of magnetic flux leakage is ignored, the magnitude of magnetic force can be simplified as the following expression [23]:

$$
F=\frac{B^{2} S}{2 \mu_{0}}
$$

The magnetic induction intensity $B$ in (1) is given by

$$
B=\frac{\mu_{0} n i}{2 e}
$$

where $S$ is the area of magnetic pole, $\mu_{0}$ is the permeability of vacuum, equal to $4 \times 10^{-7}$,e is the thickness of air gap, $n$ is the coil turn, and $i$ is the magnitude of current. 
The U-shaped magnet core is applied in the device, so the resultant magnetic force is

$$
F=\frac{B^{2} S}{\mu_{0}}=\mu_{0} n^{2} S \cdot \frac{i^{2}}{e^{2}}
$$

After the structure of electromagnet is designed, the parameters $S$ and $n$ are constant, and the thickness of air gap $e$ is basically unchanged, so the magnetic force $F$ will mainly depend on the square of the current as $i^{2}$. According to (3) and parameters in Table 1 , the maximum magnetic force of the device is $305.6 \mathrm{~N}$.

The alternating load needs to be applied in the dynamic measurement of spindle. If the current of coil is $i=I \sin w t$, the magnetic induction intensity will be $B=B_{0} \sin w t$. So the magnetic force is

$$
\begin{aligned}
F & =\frac{B^{2} S}{\mu_{0}}=\frac{B^{2} S}{\mu_{0}} \sin ^{2} w t \\
& =\frac{B_{0}^{2} S}{\mu_{0}}(1-\cos 2 w t)=F_{0}-F_{2} \cos 2 w t .
\end{aligned}
$$

Because magnetic force can only be an attractive force and its frequency is the double of the current, a direct current must be provided to magnetize the iron core beforehand, which is able to improve the harmonic characteristics of magnetic force. Therefore, the current is $i=I_{0}+I \sin w t$, and the magnetic induction intensity is $B=B_{0}+B_{1} \sin w t$. The magnetic force turns into

$$
\begin{aligned}
F & =\frac{S}{\mu_{0}}\left(B_{0}+B_{1} \sin w t\right)^{2} \\
& =\frac{S}{\mu_{0}}\left\{\left(B_{0}^{2}+\frac{B_{1}^{2}}{2}\right)+2 B_{0} B_{1} \sin w t-\frac{B_{1}}{2} \cos 2 w t\right\} \\
& =F_{0}+F_{1} \sin w t-F_{2} \cos 2 w t .
\end{aligned}
$$

If the frequency of magnetic force is to be $w$, the condition of $B_{0} \geq B_{1}$ should be met to make $F_{0} \geq F_{1}$. However, oversize $B_{0}$ may lead to the saturation of magnetic flux.

As for traditional electromagnetic loading device, it is difficult to measure magnetic force directly; hence, the indirect method of measuring magnetic flux is presented [23-26]. A specific coil is applied to induce the change of magnetic flux to generate current; then the induced current needs to be calibrated with exciting force. The inductive electromotive force in coil can be expressed as (6) on the basis of electromagnetic theory:

$$
e=-n S \frac{d B}{d t}=-n S \frac{d\left(B_{0}+B_{1} \sin w t\right)}{d t}=-n S B_{1} \cos w t .
$$

There is only the alternating part $B_{1} \sin w t$ to be measured, and the constant $B_{0}$ is neglected; therefore the constant force $F_{0}$ and the double frequency exciting force in (5)

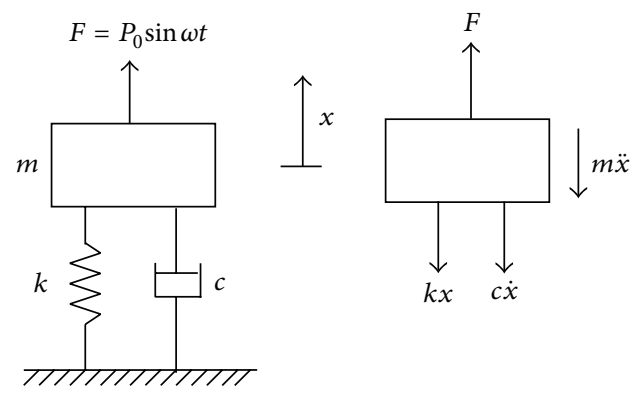

FIGURE 4: Mechanical model of electromagnetic vibration exciter.

cannot be measured availably. Furthermore, the calibration of dynamic force with induced current is difficult and inefficient. So, the method is unsuitable to measure dynamic force in the measurement of spindle stiffness.

A new method of measuring force is presented as Figure 2, which is implemented by measuring the reacting force of tool countering electromagnet using the strain type force sensors. So it is able to measure not only steady loading force but also the transient loading force exactly.

\section{Mechanical Characteristic Analysis of Loading Device}

3.1. Mechanical Model. In the basic loading device composed of electromagnet, force sensors, and support table, the thin-walled structure of force sensors causes deficient stiffness. Because the stiffness and mass of magnet are much larger than force sensors, the magnet can be simplified as a mass block and the force sensors as a springdamper component. Therefore, the loading device can be regarded as a single freedom of spring mass system shown as Figure 4.

According to the force analysis of mechanical model, the motion differential equation is obtained as follows:

$$
\begin{aligned}
& m \ddot{x}+c \dot{x}+k x=P_{0} \sin \omega t, \\
& \text { Namely: } F_{a}+F_{c}+F_{e}=F,
\end{aligned}
$$

where $F$ is the exciting force, $F_{e}$ is the force measured by force sensors, $F_{a}$ is the inertia force of electromagnet, $F_{c}$ is the damping force, $m$ is the mass of electromagnet, $k$ is the stiffness of force sensors, and $c$ is the damping coefficient.

By (8), the force measured by force sensors as $F_{e}=F-$ $F_{a}-F_{c}$ is the resultant force of exciting force, inertia force, and damping force, instead of the pure exciting force. Therefore, the exciting force needs to be calibrated using inertia force and damping force.

3.2. Dynamic Calibration of Exciting Force. As for static loading, the force acting on the electromagnet is constant, and the force measured by sensors is not affected by inertia force and damping force. However, the effect of inertia force and damping force must be eliminated in dynamic loading. 


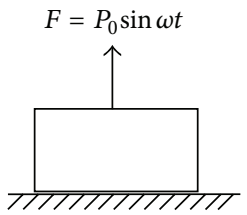

(a) Without vibration isolation

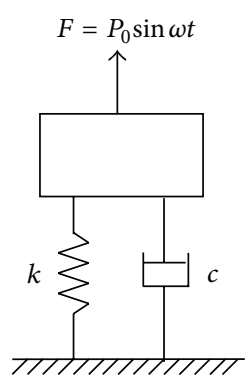

(b) Vibration isolation

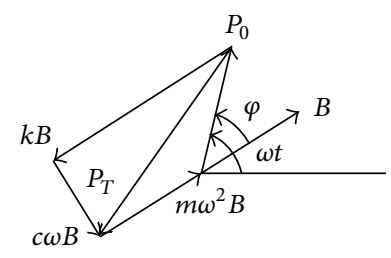

(c) Rotation vector diagram

FIGURE 5: Force transmissibility of electromagnetic vibration exciter.

A method of calibrating by force transmissibility is presented based on the principle of vibration isolation.

In Figure 5, the force acting on support table is $P_{0}$ when being without vibration isolation, but it changes to $P_{T}$ after vibration isolation. The force $P_{T}$ is given by

$$
P_{T}=\sqrt{(k B)^{2}+(c \omega B)^{2}}=k B \sqrt{1+(2 \zeta \lambda)^{2}},
$$

where $\lambda=\omega / \omega_{n}$ is the frequency ratio, $\zeta=c / 2 \omega_{n} m$ is the relative damping coefficient, $B=\left(P_{0} / k\right)\left(1 / \sqrt{\left(1-\lambda^{2}\right)^{2}+(2 \zeta \lambda)^{2}}\right)$ is the vibration amplitude of electromagnet, and $\omega_{n}=\sqrt{k / m}$ is the natural frequency.

According to the rotation vector diagram, the force transmissibility from electromagnet to support table is

$$
T_{f}=\frac{P_{T}}{P_{0}}=\sqrt{\frac{1+(2 \zeta \lambda)^{2}}{\left(1-\lambda^{2}\right)^{2}+(2 \zeta \lambda)^{2}}},
$$

while the force transmissibility from electromagnet to force sensors is

$$
T_{S}=\frac{1}{\sqrt{\left(1-\lambda^{2}\right)^{2}+(2 \zeta \lambda)^{2}}}
$$

Therefore, the real exciting force can be simulated by the formula $F=F_{e} / T_{S}$ as long as the force transmissibility is obtained.

The method of obtaining force transmissibility is shown as Figure 6.

The electromagnet is excited by an electrodynamics' vibrator using the method of sine sweep. The force on the right side of electromagnet is measured by the sensor $B$, and it is equivalent to the force $F$. The force measured by the sensor $A$ is equivalent to the force $F_{e}$. Then the force transmissibility in a certain frequency can be obtained as $T_{S}=F_{e} / F$, which consists of amplitude frequency response curve and phase frequency response curve.

The force transmissibility of the electromagnet of the loading device is measured as shown in Figure 7, which shows that there are two obvious modes at $251 \mathrm{~Hz}$ and $424 \mathrm{~Hz}$.

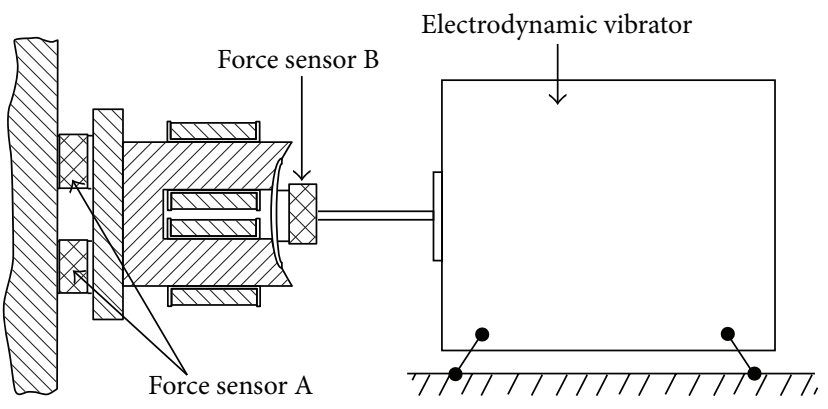

FIgURE 6: Test methods based on force transmissibility.
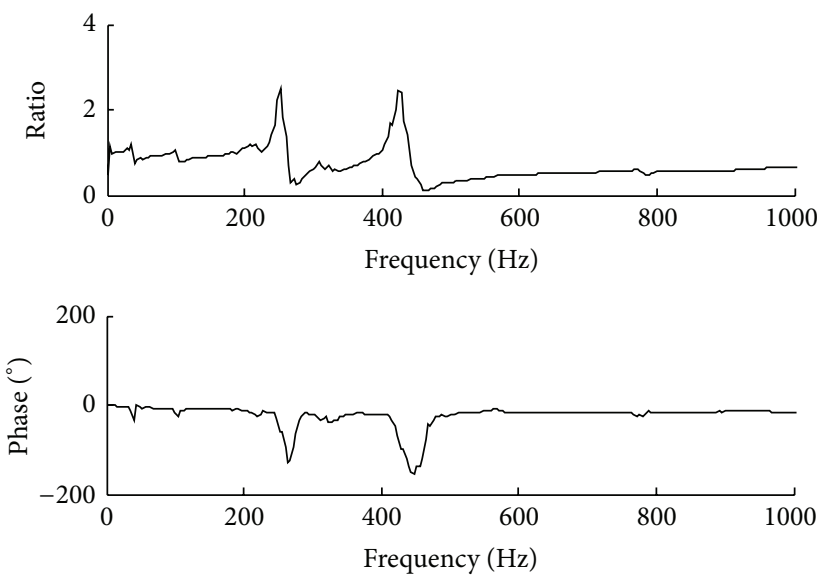

FIGURE 7: Force transmissibility of electromagnetic vibration exciter.

\section{Measurement of Dynamic Stiffness}

The dynamic stiffness of high speed spindle reflects the ability to resist deformation while the spindle suffers alternating load, which is a main index of evaluating the antivibration performance of machine tools. Moreover, the dynamic stiffness of spindle under running condition is closer to the actual operating condition than stiffness, so it is more significant.

4.1. Method of Loading and Measurement. The high speed spindle installed in NC milling machine is tested by the 


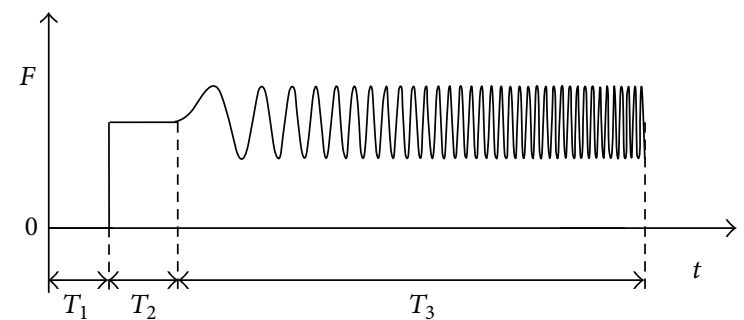

FIGURE 8: Loading scheme of dynamic stiffness measurement.

device, in order to measure the radial dynamic stiffness of spindle relative to machine working table at different speed. Some measures are taken as follows.

(1) The electromagnetic loading device is mounted on the machine working table through $\mathrm{T}$-shaped groove.

(2) The spindle needs to be warmed up by running for one hour before tests to avoid the effect of temperature.

(3) The radial load is applied to the dummy tool, when the spindle runs at different speed.

(4) The displacement sensor is placed on the other side relative to electromagnet to measure the transient deformation of spindle relative to machine working table.

(5) The exciting signal is uniform to ensure the same load applied to dummy tool.

The dynamic loading signal (shown in Figure 8) is divided into three parts: the first $T_{1}=5 \mathrm{~s}$ is a dry run process to initialize and eliminate sensors drift; the second $T_{2}=5 \mathrm{~s}$ is a process only including DC component in order to measure the stiffness of spindle; the dynamic loading is implemented in the third part $T_{3}=50 \mathrm{~s}$, which consists of the DC signal and the AC signal of the sine sweep from $0 \mathrm{~Hz}$ to $500 \mathrm{~Hz}$.

4.2. Measurement Result of Exciting Force. The spindle runs at different speeds varying from $0 \mathrm{rpm}$ to $12000 \mathrm{rpm}$, and the interval is $2400 \mathrm{rpm}$. The transient force signals measured by sensors are calibrate by the above method to obtain the actual exciting forces (shown in Figure 9). The force signals only contain AC signals while the DC signals are eliminated, so that the FRF can be obtained.

In Figure 9, taking the frequencies of $100 \mathrm{~Hz}, 200 \mathrm{~Hz}$, and $400 \mathrm{~Hz}$, for examples, the alternating exciting forces change with speed was calculated, shown in Figure 10.

According to the contrast of forces change at different speeds, the following can be seen.

(1) The magnitude of electromagnetic force reduces nonlinearly with the rise of speed. When the massive dummy tool spins rapidly in magnetic field, the dummy tool cuts magnetic induction lines, which results in intense eddy current effect at the surface of dummy tool. And the eddy current reacts to the

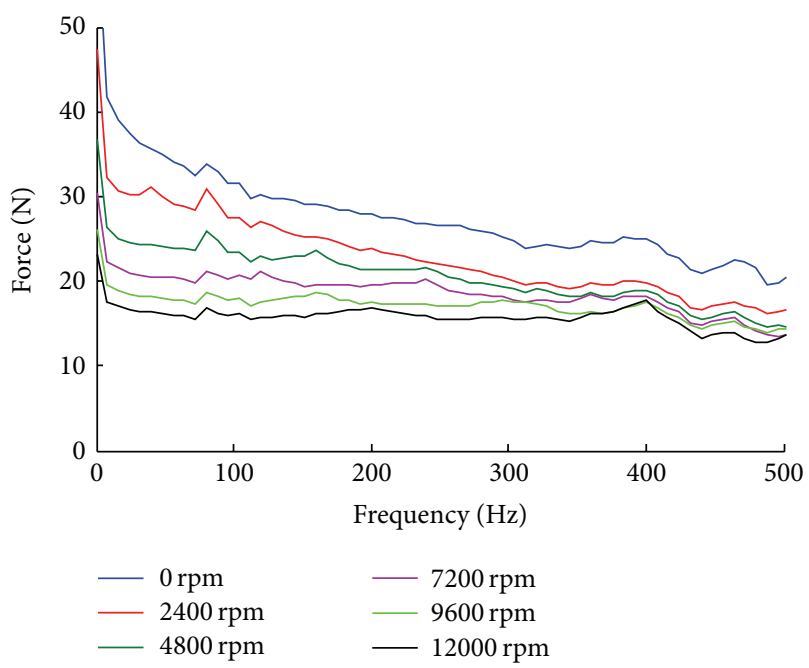

FIGURE 9: Relationship between electromagnetic force and exciting frequency at different speeds.

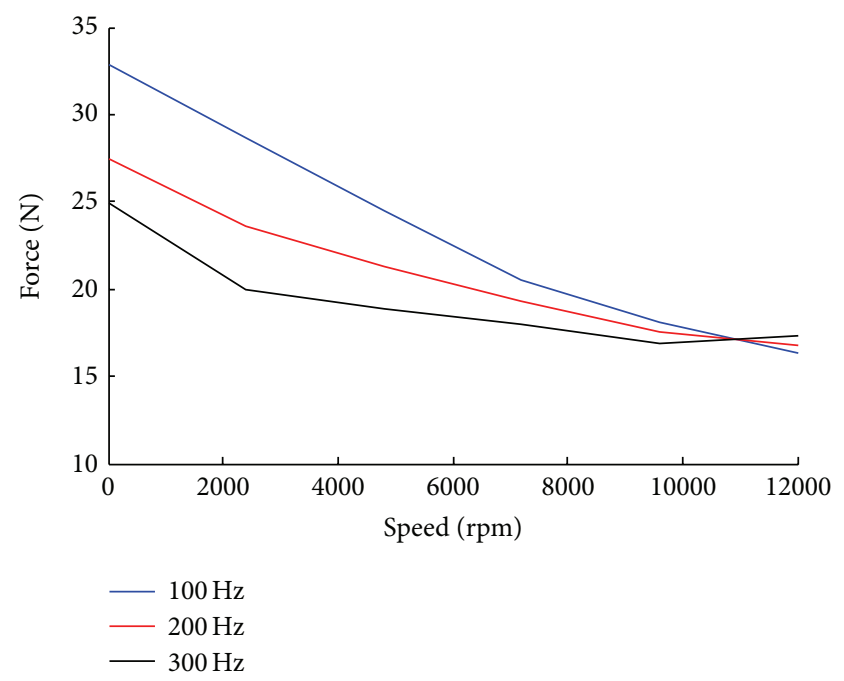

FIGURE 10: Relationship between electromagnetic force and rotational speed.

magnetic field to weaken the magnetic field intensity; therefore the electromagnetic force reduces [27-29].

(2) The magnitude of electromagnetic force reduces with the rise of frequency, which also is due to the eddy current effect and hysteresis effect.

The valid solution to the above problems is to employ thin laminated dummy tool; however it is difficult to manufacture.

4.3. Measurement of Displacement Response. The radial displacement response of spindle is measured by displacement sensor. However, the measurement result includes rotation error, roundness error, and random noise, in which the actual response signal is submerged. First, a replicate test is implemented to obtain the multiple sets of 


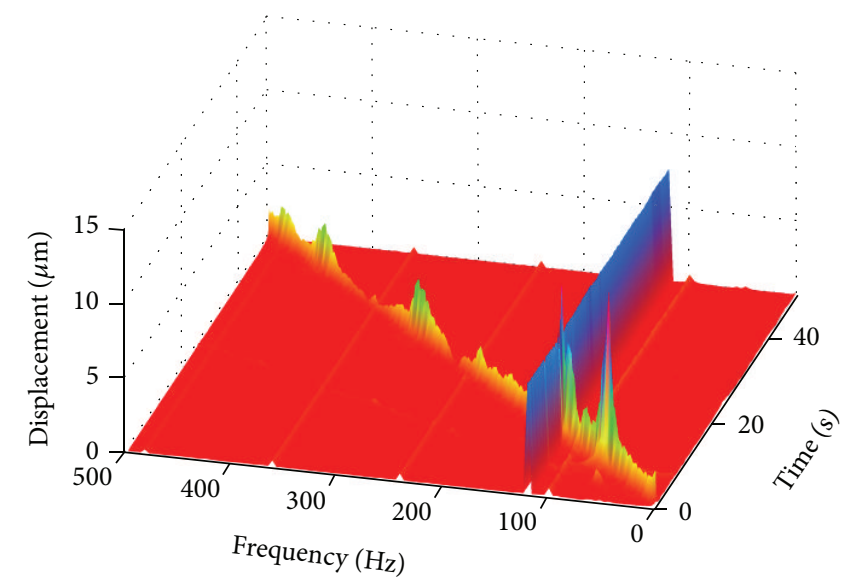

(a) Original signal

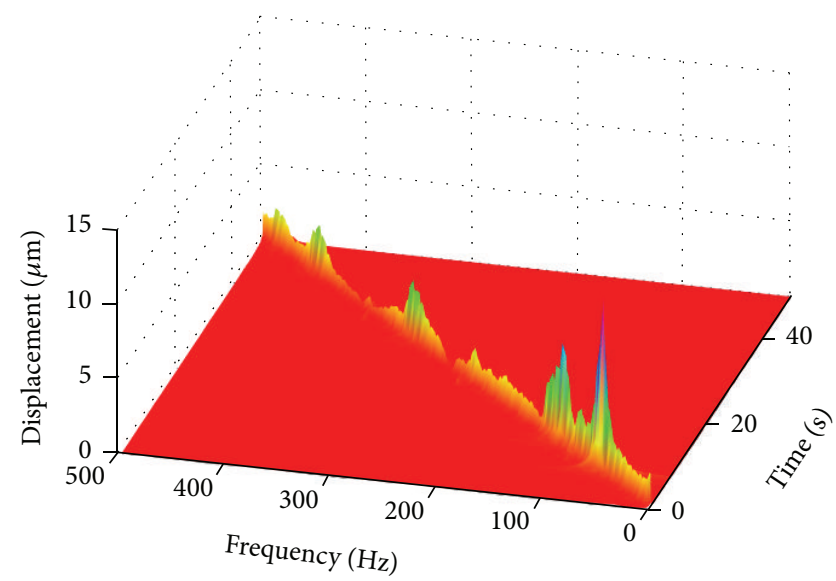

(b) Response signal

FIGURE 11: The comparison before and after the error separation processing.

experimental datum. Then, the original signals are converted to the time-frequency-domain using the short time Fourier transform (STFT). The waterfall in the case of $7200 \mathrm{rpm}$ is shown as Figure 11(a). After the overlapping portions and nonoverlapping portions between response signal and error signals are processed, respectively, by time-domain compensation and frequency-domain filtering, the rotation error and roundness error can be separated. Lastly, the multiple sets of experimental datum are averaged in time domain to eliminate the random noise. The response signal obtained by the above method is shown as Figure 11(b).

In addition, because of the method of sweep excitation, the peak holding algorithm needs to be implemented to the actual response signals of frequency-domain. The known sweep excitation from $0 \mathrm{~Hz}$ to $500 \mathrm{~Hz}$ is finished in $50 \mathrm{~s}$, and the sampling frequency is $4096 \mathrm{~Hz}$, so the relationship between time and frequency is decided. To ensure the actual amplitude of response signals, the frequency resolution of $4 \mathrm{~Hz}$ is necessary. If we want to improve the frequency resolution, the time of sweep excitation must be increased accordingly.

The response signals of different speeds are shown in Figure 12.

The variation of displacement response at different speeds is basically consistent, which verifies the availability of the loading and measurement as well as the error separation processing. Due to the difference of loading applied on spindle, it is insignificant to compare the data with each other. Therefore, the FRF of spindle is necessary.

\section{Analysis for Dynamic Stiffness}

5.1. FRF of Spindle Relative to Working Table. The FRF curves are computed using the data of exciting forces (Figure 9) and displacement responses (Figure 12).

Figure 13 consists of amplitude frequency response curve and phase frequency response curve, which shows the consistent variation in amplitude and phase with the frequency.

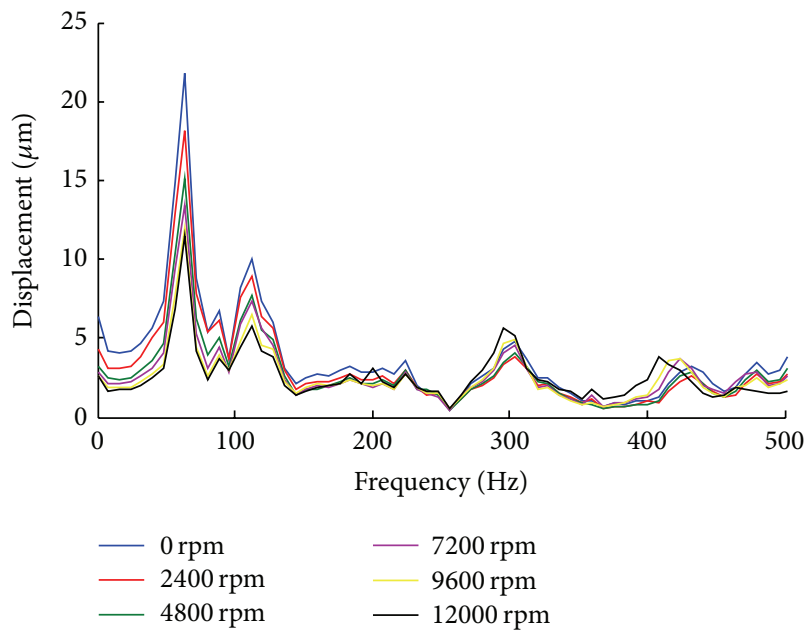

FIGURE 12: Relationship between displacement response and exciting frequency at different speeds.

Nevertheless, there are differences for the dynamic flexibility (displacement/force).

5.2. Analysis for Dynamic Stiffness and Damping Ration. The FRF curves (Figure 13) show that the dynamic flexibility varies from $0.091 \mu \mathrm{m} / \mathrm{N}$ to $0.7003 \mu \mathrm{m} / \mathrm{N}$, so the corresponding variation range of dynamic stiffness is $1.43 \sim 52.37 \mathrm{~N} / \mu \mathrm{m}$. However, researchers are usually interested in the minimum stiffness at resonant position or other modal parameters instead of the whole frequency range. In order to reflect the stiffness variation with speeds, the dynamic stiffness at $60 \mathrm{~Hz}, 112 \mathrm{~Hz}$, and $300 \mathrm{~Hz}$, which are the frequencies of the first three orders' resonance, are computed. Besides, the relevant damping ratio is also obtained, respectively, by fitting using the rational fraction polynomial method (RFP) in the frequency domain.

Static stiffness, which reflects the ability to resist deformation, is the foundation of dynamic stiffness and determines 

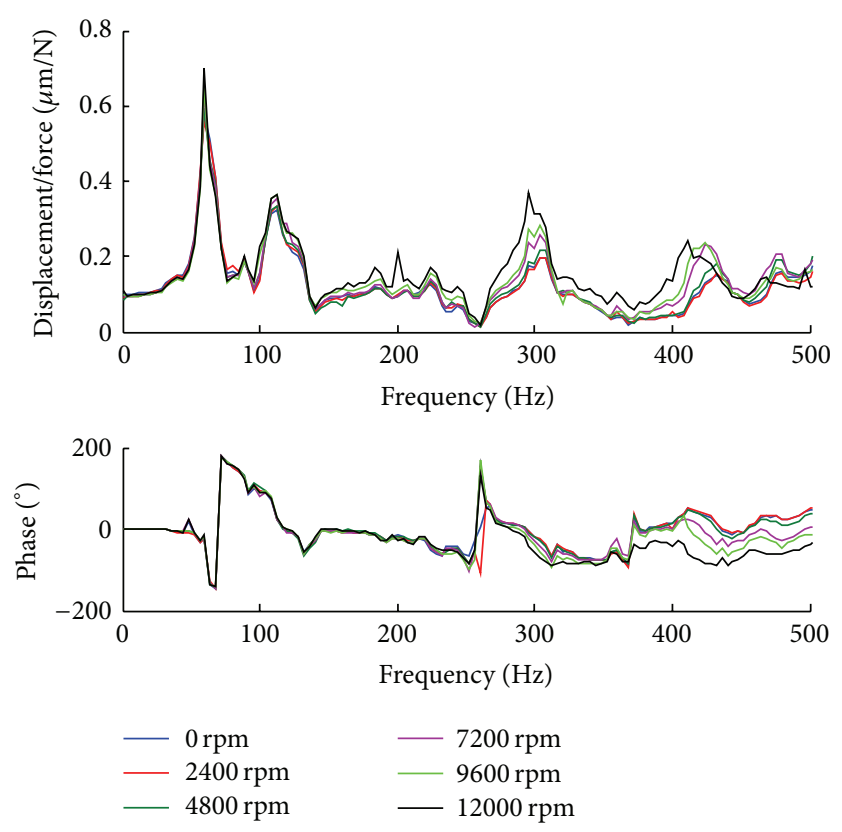

FIGURE 13: Frequency response curves of spindle displacement relative to machine working table at different speeds.

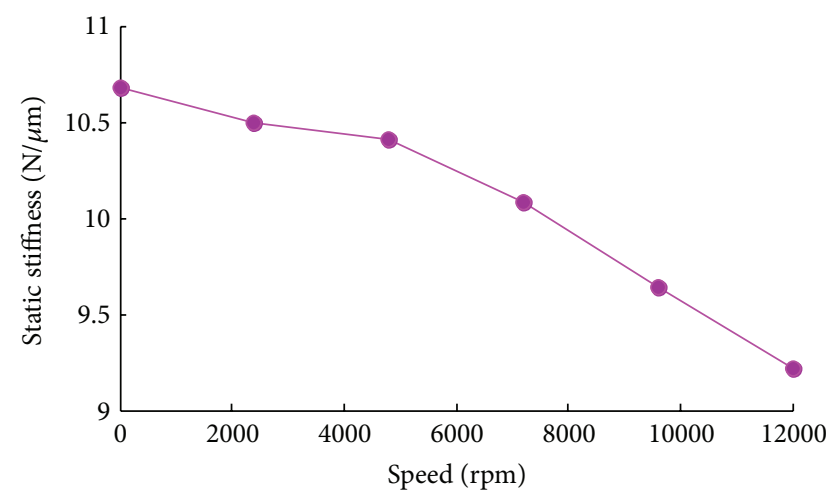

Figure 14: Static stiffness at different speeds.

the basic parameter $K$ of dynamic stiffness. Therefore, it is necessary to analyze static stiffness ahead of dynamic stiffness. According to Figure 13, the relationship between the static stiffness of spindle and rotating speeds can be obtained at the point of $0 \mathrm{~Hz}$.

Figure 14 shows that the static stiffness of spindle relative to working table trends down with speed increase. The static stiffness of static spindle is $10.68 \mathrm{~N} / \mu \mathrm{m}$, while it drops down to $9.21 \mathrm{~N} / \mu \mathrm{m}$ when the speed reaches $12000 \mathrm{rpm}$.

At different rotating speeds, the changes of dynamic stiffness and the corresponding damping ratio at the frequencies of the first three orders resonance are shown in Figure 15.

With the increase of spindle speed, the dynamic stiffness of spindle at the first three orders' resonance decreases entirely, especially the third order $300 \mathrm{~Hz}$. Compared to the static stiffness of spindle, the dynamic stiffness has the similar trend, but an obvious smaller magnitude. The test results show that both static and dynamic stiffness of spindle are affected by speed. The higher the speed is, the lower the stiffness is. The rotor of spindle is supported by angular contact ball bearings. With the increased spindle speed, the centrifugal force will push the bearing balls outward, which soften the bearing because of the contact area decrease between the bearing balls and inner ring. The stiffness of spindle mainly depends on its support bearings, therefore the static and dynamic stiffness of spindle decline.

Similar to the changes of dynamic stiffness, the damping ratios at the first three orders' resonance are also reduced with the increase of spindle speed. The reason may be due to two parts: (1) at higher speed, the centrifugal force results in the more inadequate bearing lubrication and thinning lubricant film, which is the key to the reduction of damping ratio; (2) the reduction of contact area between the bearing balls and inner ring results in the lower power consumption of vibration.

Although the variation regularity of dynamic stiffness and damping ratio is the same as the rotational speed is raised, the extent of reduction is not regular. The reduction of stiffness is largest at $300 \mathrm{~Hz}$ while the damping ratio is at $60 \mathrm{~Hz}$. It may depend on the characteristic of the measured machine tool. Furthermore, the deficiency of the research is that the relationship between the resonant frequency and rotating speeds cannot be identified due to the large frequency resolution.

\section{Conclusion}

To overcome the problems of ineffective loading and dynamic performance measurement of motorized spindle, this paper describes a method of noncontact electromagnetic loading and measuring. This approach provides a method of simulating cutting load by using electromagnetic force, and the exciting force is measured by force sensors. After the high speed spindle of NC milling machine is tested, the FRF curves of the spindle relative to machine table are collected at $0 \sim 12000 \mathrm{rpm}$; then the relationships between stiffness and speeds as well as between damping ratio and speeds are obtained. According to the test experiment, the conclusions are as follows.

(1) The method of electromagnetic loading can excite effectively the spindle at high speed rotating by using sweep excitation, but the electromagnetic force will reduce nonlinearly with the rise of speed or sweep frequency because of eddy current effect.

(2) The dynamic calibration of loading force is presented based on the principle of vibration isolation, and the steady and transient loading force can be measured directly and exactly. The displacement response can be extracted by error separation processing from the original signals measured by displacement sensors. Then the FRF curves can be obtained.

(3) The results show that not only the radial static and dynamic stiffness of the spindle but also the damping ratio declines obviously with the increase of spindle 

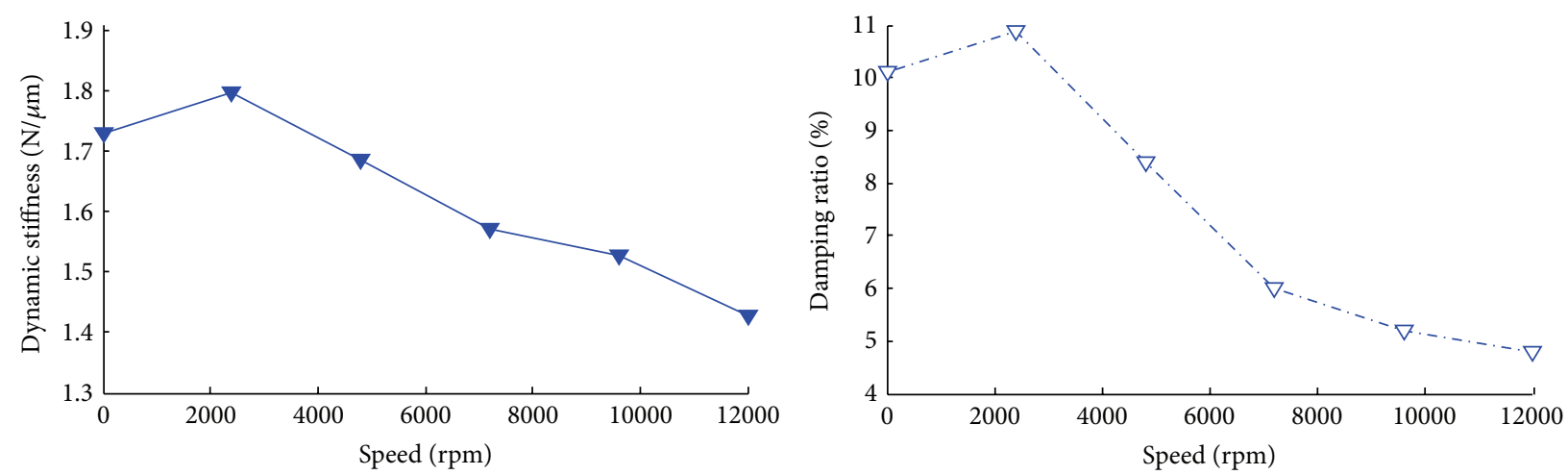

(a) Dynamic stiffness and damping ratio of $60 \mathrm{~Hz}$
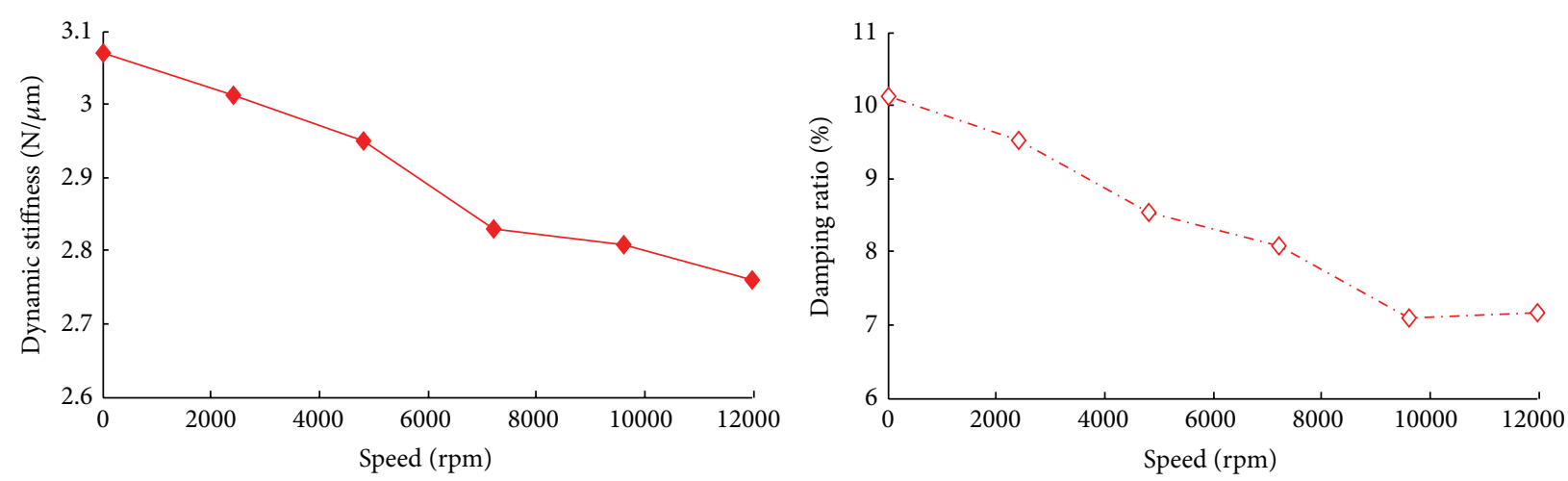

(b) Dynamic stiffness and damping ratio of $112 \mathrm{~Hz}$
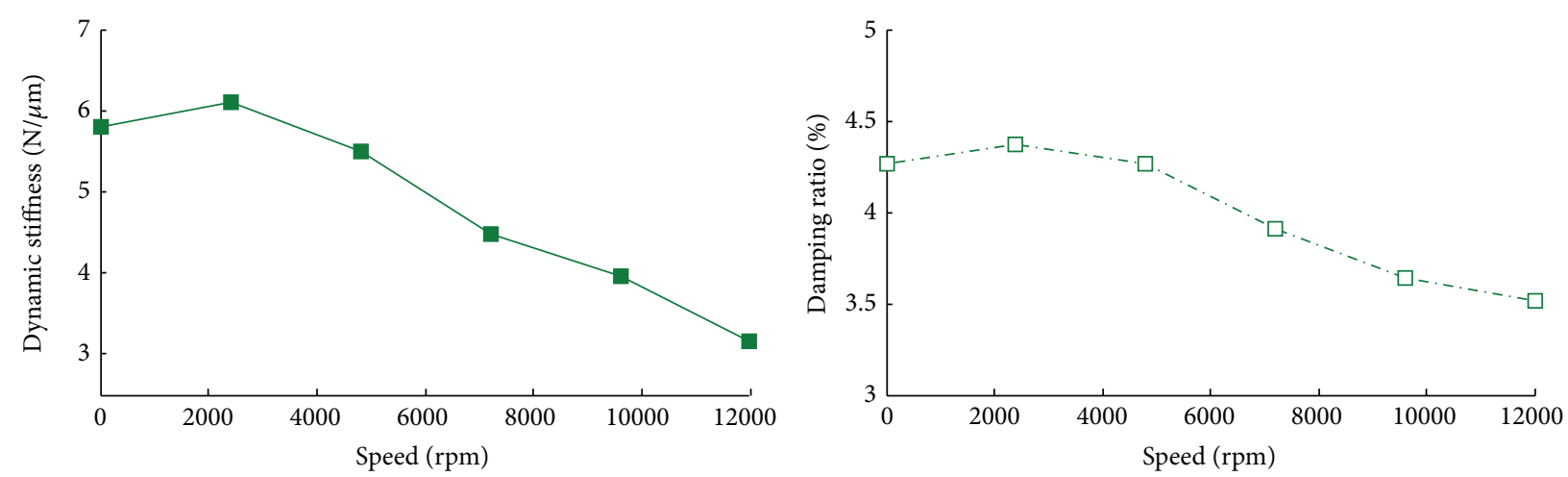

(c) Dynamic stiffness and damping ratio of $300 \mathrm{~Hz}$

FIGURE 15: Dynamic stiffness and damping ration of the first three orders resonance at different speeds.

speed. The reason may be that the centrifugal force results in the softening of the bearing and poor lubrication.

\section{Conflict of Interests}

The authors declare that there is no conflict of interests regarding the publication of this paper.

\section{Acknowledgments}

The authors wish to acknowledge the supports by INVT electric machinery (Xian) Co., Ltd, and $\mathrm{m}+\mathrm{p}$ international
Mess- und Rechnertechnik GmbH. The result work is supported by Program for Changjiang Scholar and Innovation Research Team in University (no. IRT1172) and the National Key Technology Support Program (no. 2012BAF01B02).

\section{References}

[1] E. Abele, Y. Altintas, and C. Brecher, "Machine tool spindle units," CIRP Annals-Manufacturing Technology, vol. 59, no. 2, pp. 781-802, 2010.

[2] W. R. Wang and C. N. Chang, "Dynamic analysis and design of a machine tool spindle-bearing system," Journal of Vibration and Acoustics, Transactions of the ASME, vol. 116, no. 3, pp. 280-285, 1994. 
[3] H. D. Nelson and J. M. McVaugh, "The dynamics of rotorbearing systems using finite elements," Journal of Engineering for Industry, vol. 98, no. 2, pp. 593-600, 1976.

[4] E. S. Zorzi and H. D. Nelson, "Finite element simulation of rotor-bearing systems with internal damping," American Society of Mechanical Engineers, vol. 99, no. 1, pp. 71-76, 1976.

[5] G. L. Xiong, J. M. Yi, C. Zeng, H. K. Guo, and L. X. Li, "Study of the gyroscopic effect of the spindle on the stability characteristics of the milling system," Journal of Materials Processing Technology, vol. 138, no. 1-3, pp. 379-384, 2003.

[6] Y. Z. Cao and Y. Altintas, "A general method for the modeling of spindle-bearing systems," Journal of Mechanical Design, vol. 126, no. 6, pp. 1089-1104, 2004.

[7] V. Gagnola, P. Ray, B. C. Bouzgarrou, and C. Barra, "Modelling approach for a high speed machine tool spindle-bearing system," in Proceedings of the ASME International Design Engineering Technical Conferences and Computers and Information in Engineering Conference (DETC '05), pp. 305-313, Long Beach, Calif, USA, September 2005.

[8] C.-W. Lin, J. F. Tu, and J. Kamman, "An integrated thermomechanical-dynamic model to characterize motorized machine tool spindles during very high speed rotation," International Journal of Machine Tools and Manufacture, vol. 43, no. 10, pp. 1035-1050, 2003.

[9] H. Q. Li and Y. C. Shin, "Analysis of bearing configuration effects on high speed spindles using an integrated dynamic thermomechanical spindle model," International Journal of Machine Tools and Manufacture, vol. 44, no. 4, pp. 347-364, 2004.

[10] M. Rantatalo, J. O. Aidanpää, B. Göransson, and P. Norman, "Milling machine spindle analysis using FEM and non-contact spindle excitation and response measurement," International Journal of Machine Tools and Manufacture, vol. 47, no. 7-8, pp. 1034-1045, 2007.

[11] W. G. Li, H. B. Pu, Q. Q. Liu, G. Chen, and S. Zhang, "ANSYSbased dynamic analysis of high-speed motorized spindle," in Proceedings of the International Conference on Computer Engineering and Technology, pp. 336-340, 2009.

[12] D. Liu, H. Zhang, Z. Tao, and Y. Su, "Finite element analysis of high-speed motorized spindle based on ANSYS," The Open Mechanical Engineering Journal, vol. 5, no. 1, pp. 1-10, 2011.

[13] A. S. Delgado, E. Ozturk, and N. Sims, "Analysis of nonlinear machine tool dynamic behavior," in Proceedings of the 5th Manufacturing Engineering Society International Conference, Zaragoza, Spain, June 2013.

[14] T. Holkup, H. Cao, P. Kolár, Y. Altintas, and J. Zelený, "Thermomechanical model of spindles," CIRP Annals-Manufacturing Technology, vol. 59, no. 1, pp. 365-368, 2010.

[15] V. Gagnola, B. C. Bouzgarrou, P. Ray, and C. Barra, "Modelbased chatter stability prediction for high-speed spindles," International Journal of Machine Tools and Manufacture, vol. 47, no. 7-8, pp. 1176-1186, 2007.

[16] I. Zaghbani and V. Songmene, "Estimation of machine-tool dynamic parameters during machining operation through operational modal analysis," International Journal of Machine Tools and Manufacture, vol. 49, no. 12-13, pp. 947-957, 2009.

[17] A. Albrecht, S. S. Park, Y. Altintas, and G. Pritschow, "High frequency bandwidth cutting force measurement in milling using capacitance displacement sensors," International Journal of Machine Tools \& Manufacture, vol. 45, no. 9, pp. 993-1008, 2005.

[18] E. Ozturk, U. Kumar, S. Turner, and T. Schmitz, "Investigation of spindle bearing preload on dynamics and stability limit in milling," CIRP Annals Manufacturing Technology, vol. 61, no. 1, pp. 343-346, 2012.

[19] C.-H. Cheng, T. L. Schmitz, and G. S. Duncan, "Rotating tool point frequency response prediction using RCSA," Machining Science and Technology, vol. 11, no. 3, pp. 433-446, 2007.

[20] J.-S. Chen and K.-W. Chen, "Bearing load analysis and control of a motorized high speed spindle," International Journal of Machine Tools and Manufacture, vol. 45, no. 12-13, pp. 14871493, 2005.

[21] T. Tsuneyoshi, "Spindle preload measuement and analysis," in Proceedings of the ASPE Summer Topical Meeting on Precision Bearings and Spindles (ASPE '07), pp. 35-38, State College, Pa, USA, June 2007.

[22] M. Feng, Y. L. Zhao, W. Yang, and Y. Z. Deng, “The development of non-contact gas-loading system for stiffness measurement of high speed spindles," Machinery Design \& Manufacture, vol. 6, pp. 102-105, 2013 (Chinese).

[23] A. W. Kwiatkowski, "A magnetic vibrator for the determination of machine tool dynamic characteristics," in Proceedings of the 9th MTDR Conference, vol. 1, pp. 571-590, Pergamon Press, Oxford, UK, 1968.

[24] I. Chowdhury, M. M. Sadek, and S. A. Tobias, "Determination of the dynamic characteristics of machine tool structures," Proceedings of the Institution of Mechanical Engineers, vol. 184, pp. 943-960, 1969.

[25] T. Yamazaki, A. Matsubara, T. Fujita, T. Muraki, K. Asano, and K. Kawashima, "Measurement of spindle rigidity by using a magnet loader," Journal of Advanced Mechanical Design, Systems and Manufacturing, vol. 4, no. 5, pp. 985-994, 2010.

[26] A. Matsubara, T. Yamazaki, and S. Ikenaga, "Non-contact measurement of spindle stiffness by using magnetic loading device," International Journal of Machine Tools \& Manufacture, vol. 71, pp. 20-25, 2013.

[27] M. Ahrens and L. Kucera, "Analytical calculation of fields, forces and losses of a radial magnetic bearing with a rotating rotor considering eddy currents," in Proceedings of the 5th International Symposium on Magnetic Bearings, pp. 308-313, Kanazawa, Japan, August 1996.

[28] Y. H. Sun and L. Yu, "Analytical method for eddy current loss in laminated rotors with magnetic bearings," IEEE Transactions on Magnetics, vol. 38, no. 2, pp. 1341-1347, 2002.

[29] K. Muramatsu, T. Shimizu, A. Kameari et al., "Analysis of eddy currents in surface layer of laminated core in magnetic bearing system using leaf edge elements," IEEE Transactions on Magnetics, vol. 42, no. 4, pp. 883-886, 2006. 

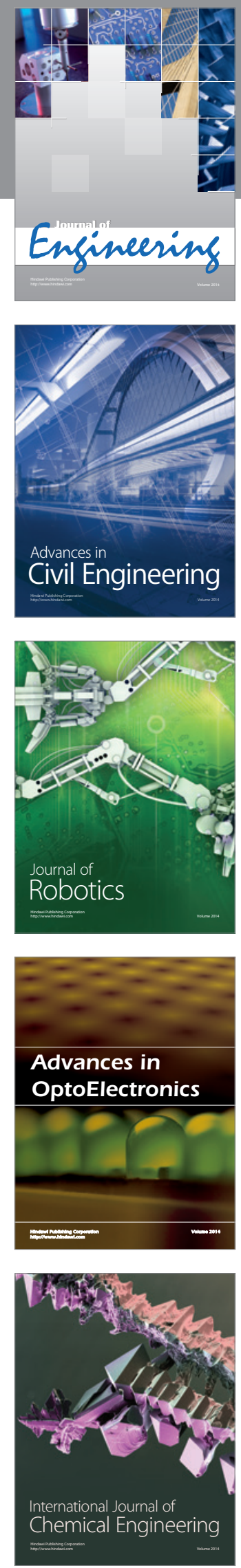

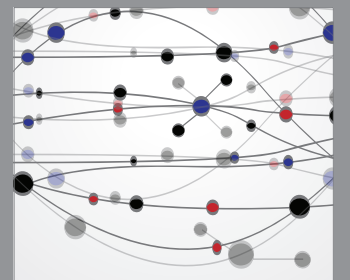

The Scientific World Journal
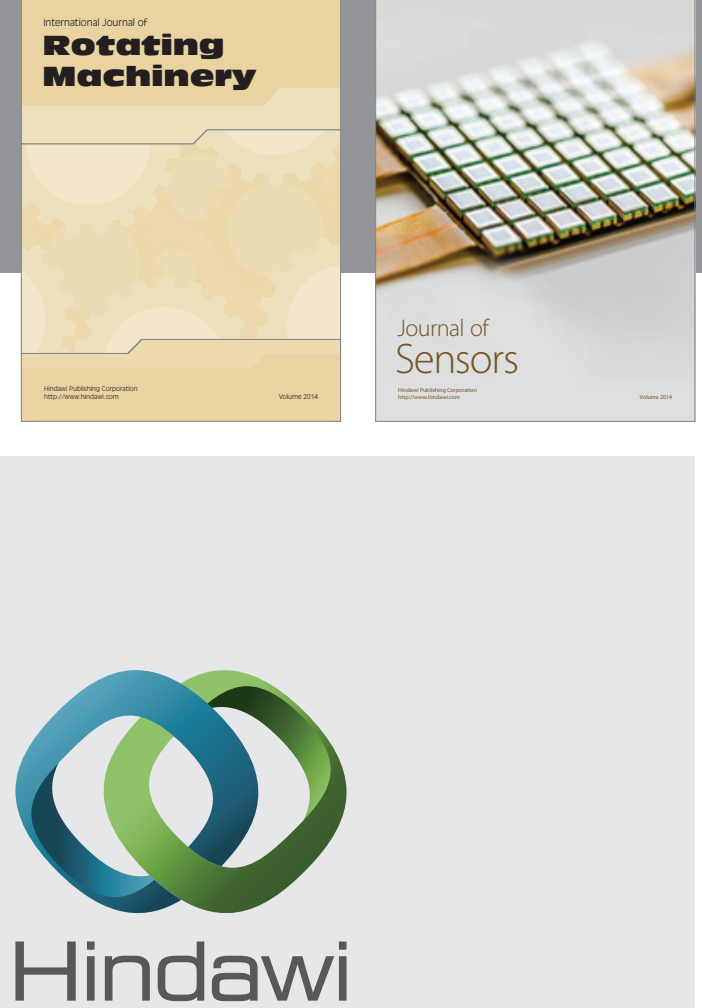

Submit your manuscripts at http://www.hindawi.com
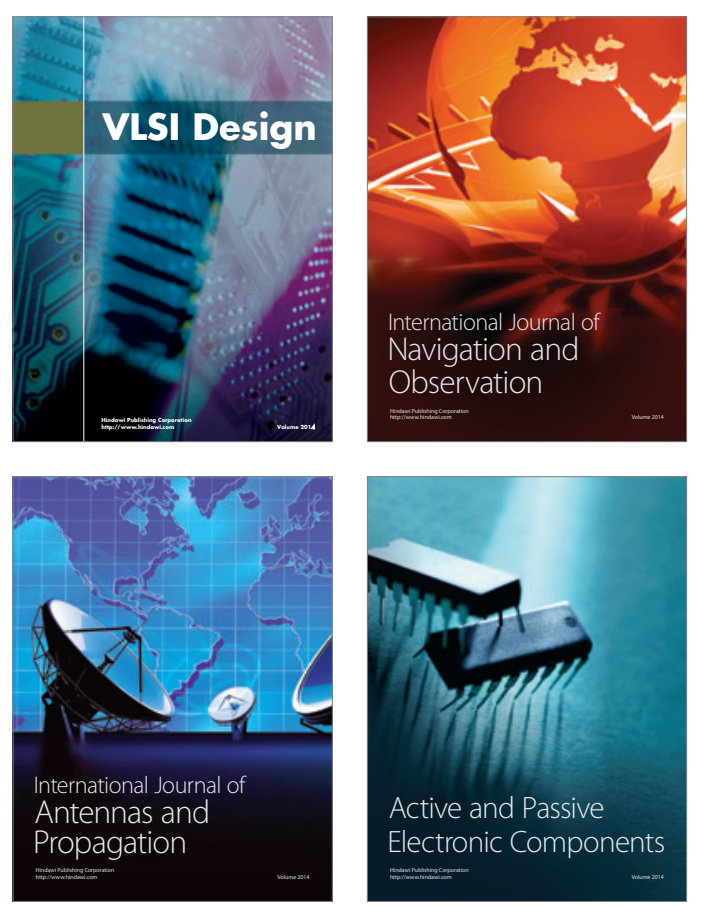
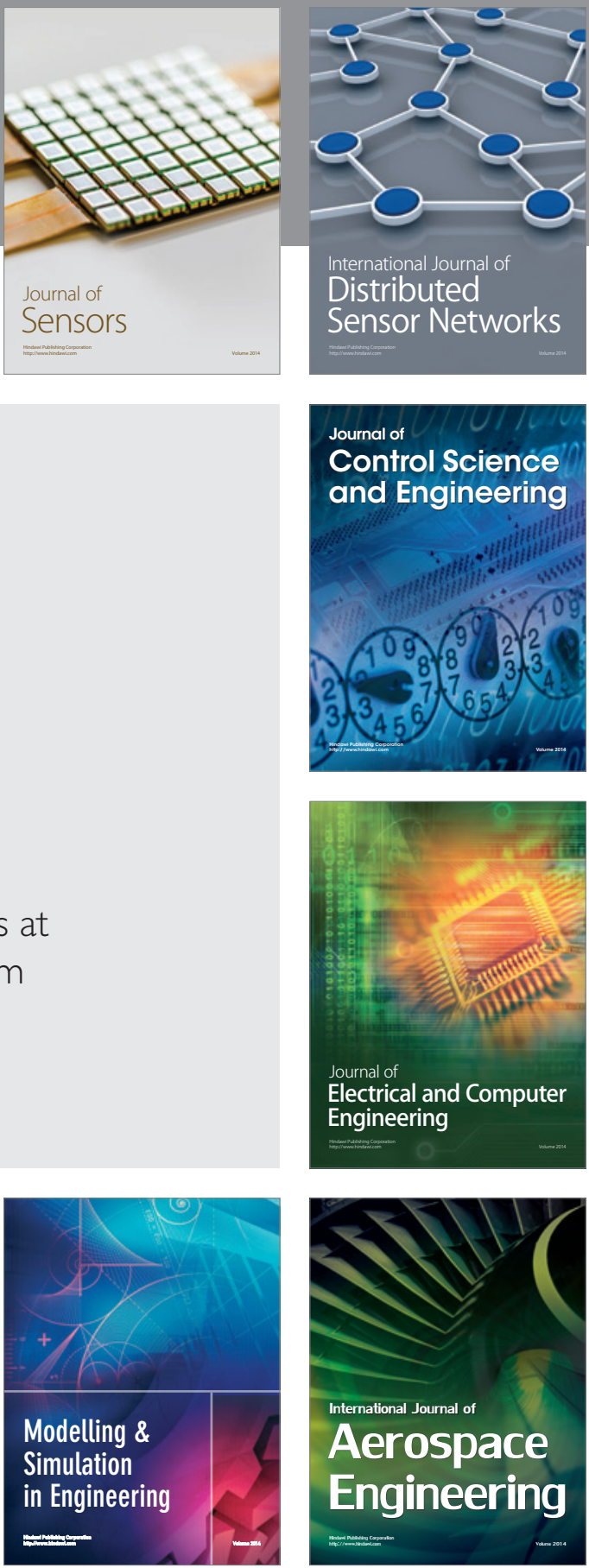

Journal of

Control Science

and Engineering
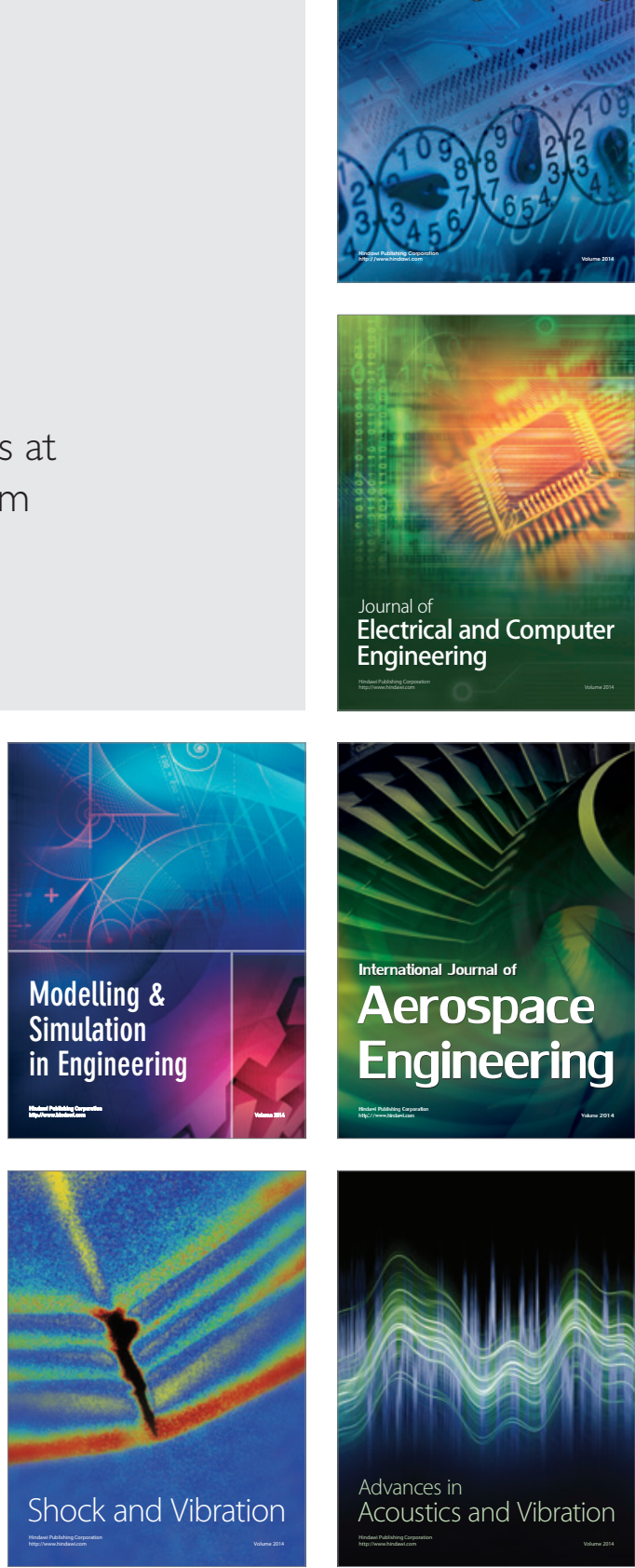